\title{
GnRH agonist versus GnRH antagonist in in vitro fertilization and embryo transfer (IVF/ET)
}

\author{
Raffaella Depalo ${ }^{1 *}$, K Jayakrishan², Gabriella Garruti ${ }^{3}$, Ilaria Totaro ${ }^{1}$, Mariantonietta Panzarino', \\ Francesco Giorgino ${ }^{3}$ and Luigi E Selvaggi ${ }^{1}$
}

\begin{abstract}
Several protocols are actually available for in Vitro Fertilization and Embryo Transfer. The review summarizes the main differences and the clinic characteristics of the protocols in use with GnRH agonists and GnRH antagonists by emphasizing the major outcomes and hormonal changes associated with each protocol. The majority of randomized clinical trials clearly shows that in "in Vitro" Fertilization and Embryo Transfer, the combination of exogenous Gonadotropin plus a Gonadotropin Releasing Hormone (GnRH) agonist, which is able to suppress pituitary FSH and LH secretion, is associated with increased pregnancy rate as compared with the use of gonadotropins without a $\mathrm{GnRH}$ agonist. Protocols with $\mathrm{GnRH}$ antagonists are effective in preventing a premature rise of $\mathrm{LH}$ and induce a shorter and more cost-effective ovarian stimulation compared to the long agonist protocol. However, a different synchronization of follicular recruitment and growth occurs with GnRH agonists than with $\mathrm{GnRH}$ antagonists. Future developments have to be focused on timing of the administration of GnRH antagonists, by giving a great attention to new strategies of stimulation in patients in which radio-chemotherapy cycles are needed.
\end{abstract}

Keywords: ivf, GnRH, Oocytes, GnRH protocols

\section{Review}

Several randomized clinical trials demonstrate that in IVF-ET, the combination of exogenous gonadotropin plus Gonadotropin Releasing Hormone agonist (GnRH-a), for the suppression of pituitary FSH and LH secretion, is associated with higher pregnancy rates as compared to the use of gonadotropins without GnRH-a. The major benefits of these drugs include decreased cancellation rate through prevention of premature LH surge and luteinisation [1], enhancement of follicular recruitment, allowing the recovery of a larger number of oocytes [2], and the improvement in routine patient treatment schedule [3]. The gold standard for ovarian stimulation in young normogonadotropic women is recognized as the long protocol, starting GnRH-a in the mid luteal phase of the preceding cycle (Figure 1). A systematic overview of twenty-six trials comparing different GnRH-a protocols for pituitary

\footnotetext{
* Correspondence: ilariatotaro.it@libero.it

'Unit of Physiopathology of Human Reproduction and Gametes Cryopreservation, Department of Gynecology, Obstetric and Neonatolgy, University of Bari "Aldo Moro", Bari, Italy

Full list of author information is available at the end of the article
}

desensitization in in vitro fertilization demonstrated the superiority of the long protocol over the short and ultrashort protocols (OR 1.32 for clinical pregnancy rate per cycle started), with GnRH analogue being commenced either in follicular phase or in luteal phase [4]. GnRH-a long protocol, induces profound suppression of endogenous release of gonadotropins during the early follicular phase, allowing the early antral follicles to grow co-ordinately in response to exogenous gonadotropins to accomplish simultaneous maturation. This leads to an extended widening of the FSH window, an increased number of recruited mature follicles and a higher number of retrieved oocytes [4].

Two types of GnRH-a administration pattern can be used to lead to pituitary desensitization in the long protocol; one consisting of low dose $(0.1 \mathrm{mg})$ of GnRH-a daily and another consisting of the administration of higher doses (3.75 mg, depot) of long-acting analogues. Albuquerque et al. [5], in a meta-analysis of six randomized controlled trials (RCTs), found that pregnancy rates are similar in the long protocol using depot or daily GnRH analogues. However, the use of long-acting analogues is associated with an increasing requirement 


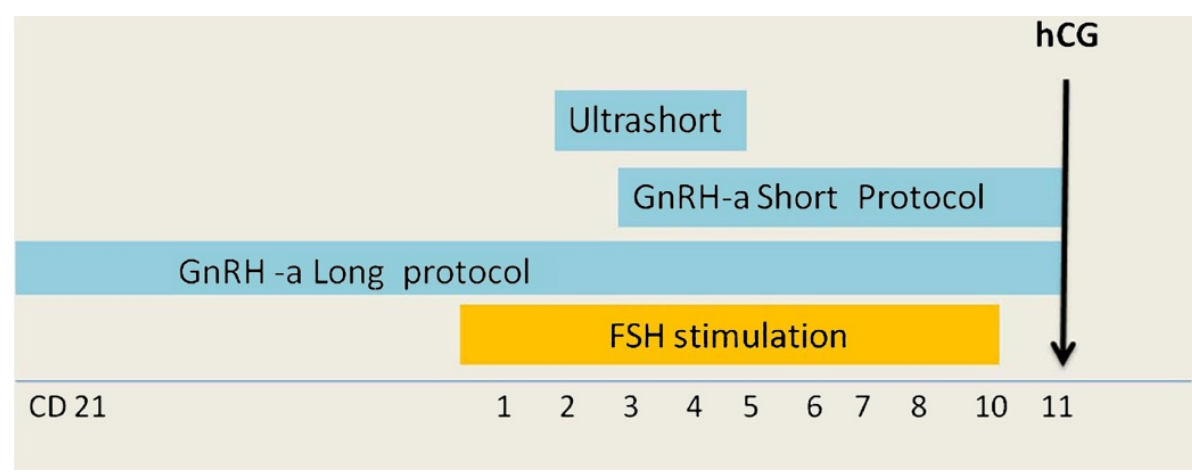

Figure $1 \mathrm{GnRH}$ agonist protocols. Long Protocol: GnRH agonist $0.1 \mathrm{mg}$ starting in follicular phase or luteal phase (Cycle Day 21) of the previuos cycle until hCG administration. Short Protocol: GnRH agonist $0.1 \mathrm{mg}$ starting on day 1 or 3 of stimulation until hCG administration. Ultrashort Protocol: GnRH agonist $0.1 \mathrm{mg}$ administered on day 2-4 of stimulation.

for gonadotropins and a longer time of ovarian stimulation compared to the daily GnRH-a low dose. In patients with normal BMI compared to over-weight patients, it was demonstrated that low doses of tryptorelin (0.05 mg, daily) are adequate to prevent a premature LH rise, resulting in reduced gonadotropin levels and increased clinical outcomes[6]. Since GnRH receptors are expressed in human ovary, it was suggested that high doses of GnRH-a may induce desensitization of ovarian receptors in normal or underweight patients. In contrast, in overweight women, increased fat mass may account for either increased steroid storage or increased peripheral conversion of androgens to estradiol (E2), thus providing a source for serum E2 levels when ovarian steroidogenesis might be suppressed [6].

The use of GnRH agonists in the long protocol is characterized by some disadvantages for the patients: a) the drawback of a long treatment period until desensitization occurs [7]; b) the increased risk of the ovarian hyperstimulation syndrome (OHSS) [8]; c) more frequent occurrence of side effects (e.g., hot flushes, headache, bleeding, and cyst development) during the desensitization period $[9,10]$.

The introduction of GnRH antagonists (GnRH-ant) in Assisted Reproductive Technologies (ART) to prevent LH surge, seemed to open up a new way towards a more "friendly IVF" [11]. Unlike the indirect pituitary suppression induced by GnRH-a, GnRH-ant administration causes immediate and dose-related inhibition of gonadotropins release by competitive occupancy of the GnRH receptors in the pituitary [12].

The use of GnRH-ant leads to a significant reduction in the duration of ovarian stimulation. $\mathrm{GnRH}$ antagonists are also not associated with acute induction of gonadotropins, which may induce cyst formation. In addition, no hot flushes are observed with $\mathrm{GnRH}$-ant because their use does not result in the profound hypo-oestrogenemia observed with GnRH-a. Finally, a reduced incidence of moderate and severe OHSS may occur while using $\mathrm{GnRH}$-ant. In a Cochrane review, Al-Inany et al. have shown that women receiving antagonists, have a significantly lower incidence of OHSS when treated with GnRh ant compared with women treated with $\mathrm{GnRh}$ agonist $(\mathrm{RD}=-0.03,95 \% \mathrm{CI}=-0.05$ to $0.02, \mathrm{P}<0.00001)[13]$

In a meta-analysis comparing $\mathrm{GnRH}$-a versus $\mathrm{GnRH}-$ ant for controlled ovarian stimulation in oocyte donors, Bodri et al. found no significant difference in the incidence of OHSS by comparing protocols with GnRH agonists versus antagonists[RR $0.61(95 \%)$ CI 0.18 to 2.15 , $\mathrm{P}=45$, heterogeneity $\mathrm{P}=45, \mathrm{I} 20 \%$ fixed effects model] [14]. Moreover, the GnRh antagonist protocol makes it possible to trigger ovulation with GnRh agonist instead of hCG, minimizing the risk of OHSS and securing the appropriate maturation of oocytes.

In a recent review, it has been demonstrated that in fresh IVF cycles with ET, no OHSS was reported after $\mathrm{GnRH}$ ant [risk difference of $5 \%$ when compared with GnRH a group (with 95\% CI: -0.07 to 0.02 )][15].

Ovulation triggering with GnRH agonist, in GnRH ant protocols is associated with the strategy to freeze all oocytes for future use, and this could be the tool towards eradication of OHSS[16]. (Written informed consent was obtained from the patient for publication of this report).

The above considerations are corroborated by recent reports indicating a classic GnRh-ant protocol where ovulation induction is carried out with GnRh agonist, associated with decreased risk of post-trigger oestradiol exposure as well as OHSS risk in women with breast cancer [17-19].

In our experience, the avoidance of an acute stimulation of endogenous gonadotropins, the short duration of treatment, and the ability to inhibit directly the premature LH surge made GnRH-ant the most appropriate regimen for ovarian stimulation, for embryo or gamete 
cryopreservation in cancer patients, prior to gonadotoxic therapy.

Two GnRH-ant regimens have been developed for controlled ovarian stimulation, involving either single administration [20] or multiple administrations [21]. (Figure 2). In the single dose protocol, the administration of a $3 \mathrm{mg}$ dose of $\mathrm{GnRH}$-ant on day 7 of the ovarian stimulation was shown to prevent a premature LH surge [22]. In the multiple dose protocol, the GnRH-ant was administered continuously until the day of hCG, and the minimal effective dose to prevent the occurrence of a premature $\mathrm{LH}$ rise was identified as $0.25 \mathrm{mg}$ of Cetrorelix [23,24]. No significant difference in pregnancy rates was shown in a randomized controlled trial which compared single injections of cetrorelix acetate $(3 \mathrm{mg})$ and a daily dose of ganirelix $(0.25 \mathrm{mg})$ in the inhibition of premature LH surge. However, the single-dose GnRH-ant protocol has the advantage to reduce the number of injections, although additional daily doses of antagonist are needed in $10 \%$ of cycles [25]. Moreover, in some cases a 3 mg-dose may result in excessive and potentially harmful suppression of endogenous LH [26].

\section{Fixed versus flexible regimen: Which is the most effective?}

Defining the most appropriate time to start cetrorelix administration has been the subject of several studies. From the physiological point of view, GnRH-ant administration should start when there is follicular development and/or production of E2 by the developing follicles which may cause a premature elevation in pituitary $\mathrm{LH}$ release, due to positive feedback mechanisms.

The most common type of treatment called fixed protocol consists of giving GnRH-ant 5 days after the stimulation with gonadotropins. However, in order to reduce the number of antagonist injections and the duration of stimulation, the flexible protocol was introduced. It consists in administering $\mathrm{GnRH}$ antagonist when the follicles reach a size of $>14 \mathrm{~mm}[27,28]$.

A meta-analysis by Al Inany [29] evaluated four RCTs $[27,28,30,31]$ that were performed to compare fixed versus flexible $\mathrm{GnRH}$-ant protocols. There was no significant statistical difference in pregnancy rate per randomized woman $(\mathrm{OR}=0.795 \% \mathrm{CI}=0.47$ to 1.05$)$, and no significant difference in the incidence of premature LH surge in both protocols.

Several studies have raised concerns regarding an unfavourable effect of late administration of GnRH-ant, either on day 6 of stimulation or later in flexible protocols. With this mode of administration, LH levels remain unsuppressed during the early follicular phase and enhance $E_{2}$ production. In the flexible protocol, high exposure of the genital tract to $\mathrm{LH}, \mathrm{E} 2$ and progesterone levels during the early follicular phase, might adversely affect the implantation rate mainly by altering endometrial receptivity, leading to a worse reproductive outcome. Kolibianakis et al., [32], in a randomized controlled trial, showed that starting the GnRH-ant either on stimulation day 1 or on stimulation day 6 resulted in equal follicular development. In addition, its use was suggested in Polycystic Ovarian Syndrome patients with high LH levels, during the follicular phase.

When analyzing follicular development and endocrine profile of patients who received their first GnRH- ant administration on day 8 or later, it was noticed that these patients had a higher number of follicles $>11$ and $<15 \mathrm{~mm}$ in diameter and high E2 and LH levels compared to patients in the fixed protocol group. This data suggests that in this flexible regimen, the cohort of

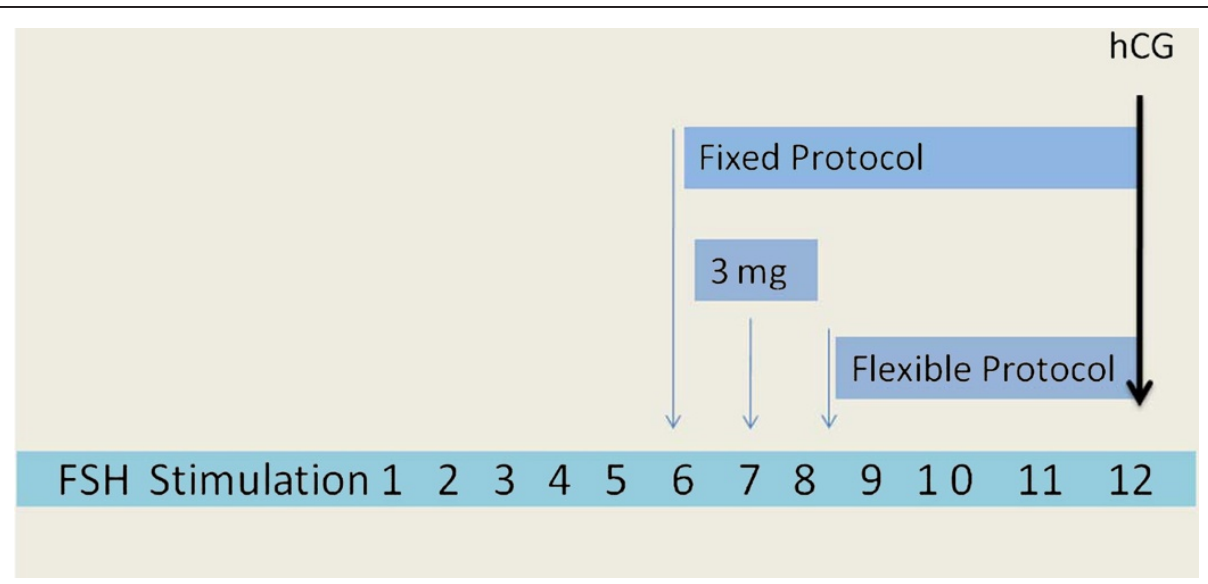

Figure 2 GnRH antagonist protocols. Fixed day 6 protocol: $0.25 \mathrm{mg} \mathrm{GnRH}$ antagonist/daily until hCG administration (Albano et al.,F\&S 1997) [23]. Single dose protocol. $3 \mathrm{mg} \mathrm{GnRH}$ antagonist at day 7 of stimulation (Olivennes et al.,HR 1998) [22]. Flexible dose protocol: $0.25 \mathrm{mg} \mathrm{GnRH}$ antagonist when follicles reach $>14 \mathrm{~mm}$ (Diedrich et al., HR 1994) [21]. 
follicles had more time to develop leading to a higher number of follicles in mid-follicular phase [28]

The optimal levels of endogenous LH in GnRH-ant cycles, are still a matter of debate. It may be assumed that the deep suppression of $\mathrm{LH}$ secretion induced by $\mathrm{GnRH}-\mathrm{a}$ administration is likely to be detrimental for the follicleoocyte complex. A low residual LH concentrations and impaired E2 secretion with increasing doses of antagonist were indeed associated with low implantation rates [24]. On the other hand, a trend towards lower pregnancy rates, was observed in patients with LH deficiency, documented by low E2:oocyte ratio, which could be explained by the endometrial impact of low LH levels [33]. On the basis of these observations, the possibility of LH supplementation in $\mathrm{GnRH}$-ant regimens was examined. Data from two randomized controlled trials showed that the addition of $75 \mathrm{IU}$ of recombinant LH to recombinant FSH at GnRHant initiation, or from initiation of stimulation, does not appear to increase pregnancy rates [34]. Similarly, no improvement in pregnancy rates could be shown by increasing the dose of HMG by 75 IU at GnRH-ant initiation [35]. Both studies show no evidence, that low endogenous LH levels after GnRH-ant initiation are associated with a decreased probability of pregnancy in IVF cycles $[31,36]$. In a third study of Baruffi et al., a meta analysis of five RCT, significantly higher serum E2 concentration and number of MII oocytes were observed in GnRh ant cycle supplemented with LH, suggesting that LH may prevent any decrease in oestradiol levels after antagonist administration even if there was no significant difference in implantation and pregnancy rates [37]. It was suggested that lower the LH levels on day 8 of stimulation for IVF, higher was the probability of pregnancy [32]. High serum LH levels at early stage of stimulation might be responsible for advanced endometrial maturation which induces an early closure of the implantation window through earlier expression of progesterone receptors in the follicular phase and downregulation of E2 receptors by the exposure to supraphysiological steroid hormone levels [30].

Huirne et al. highlighted the evidence that during GnRH-ant administration, very large changes (either increase or decrease) in LH levels, rather than absolute $\mathrm{LH}$ levels, are associated with a decreased chance of clinical pregnancy [38].

The use of oral contraceptive pill (OCP) has been considered as a mean for programming IVF cycles using GnRH-ant [33], and it has been speculated that the use of OCP pre-treatment may result in improved synchronization of the recruitable cohort of ovarian follicles. A study by Kolibianakis et al.[39] showed no significant effect of OCP pre-treatment on the probability of pregnancy in GnRH-ant cycles; however easier scheduling of the cycle, an increase of gonadotropin requirement, and a longer duration of treatment was observed with the use of OCP.
However in a recent meta-analysis, encompassing 1343 randomized patients, Griesinger et al. (2010) observed that the probability of an ongoing pregnancy per randomized woman was found to be significantly lower in patients who received OC pre-treatment. (RR 0.80, 95\% CI : 0.66 to $0.97, \mathrm{P}=0.02$ ) [40]

Finally the potential beneficial effect of GnRH-ant on pregnancy rate in intrauterine insemination(IUI) cycles, has been assessed in a recent meta-analysis conducted by Kosmas on six studies with 521 women [41]. Higher pregnancy rates were found $(16.9 \%$ in the antagonist group and $11.5 \%$ in the control group) when $\mathrm{GnRH}$-ant was administered. Moreover a trend for multiple pregnancies was also observed when GnRH-ant was administered. Increased duration for administration of gonadotropins was observed in the GnRH-ant group compared with the control-group.

\section{$\mathrm{GnRH}-\mathrm{a}$ versus $\mathrm{GnRH}$-ant regimens}

Several RCTs have been designed to compare the efficacy of the GnRH-ant with that of GnRH-a long protocol, but these studies often show conflicting results. Significantly less gonadotropin ampoule consumption and stimulation days in $\mathrm{GnRH}$-ant regimes with respect to $\mathrm{GnRH}$-a regimen $[28,42]$ was observed. No significant difference was observed in the clinical pregnancy rates and the live birth rates between the two different regimens [28]. Although a similar number of good embryos were obtained and replaced in both groups, the implantation rate and clinical and ongoing pregnancy rates tended to be lower in GnRH-ant group. The miscarriage rate however was comparable [42]. Moreover, a lower mean number of cumulus-oocyte-complexes (COC) and 2 pronuclear (PN) oocytes were found in $\mathrm{GnRH}$-ant group than in GnRH-a group [28,42].

$\mathrm{LH}$ and E2 concentrations, in early follicular phase, were higher in $\mathrm{GnRH}$-ant regime as compared with $\mathrm{GnRH}$-a regime, whereas the LH concentrations on the day of hCG were comparable in both protocols [42]. A premature $\mathrm{LH}$ rise was observed in $4.3 \%$ of $\mathrm{GnRH}$-ant patients [28] and in 3\% of GnRH-a patients [42]. OHSS grade II and III (WHO classification) was significantly higher in $\mathrm{GnRH}$-a group $(1.1 \% \mathrm{P}=0.03)$ and finally, the initiation of FSH administration in a GnRH-ant regimen was found to be cycle-dependent, making treatment planning and scheduling more difficult $[38,43]$.

Now that more than 200 papers have been published with the aim to compare the efficacy of GnRH-ant protocols with $\mathrm{GnRH}$-a long protocol, it may be time to try to close the debate. Recently, three meta-analysis have been published with the aim to compare the GnRH-ant regimens with the GnRH-a long protocol. The metaanalysis by Al Inany [13] examines the first five comparative studies of fixed GnRH-ant protocol with the 
standard GnRH-a long protocol. The OR for clinical pregnancy rate (PR) per randomized woman was 0.78 (95\% CI 0.62-0.97) in favour of agonist regimen, and the absolute treatment effect was 5\%, thus meaning that 5\% lower PR was observed with GnRH-ant regimen.

A second study by Kolibianakis et al., [39] is a metaanalytic review of 22 RCTs published as full papers in peer reviewed journals analysing a total of 3,100 patients. The primary outcome was live birth. The study showed that the probability of live birth between $\mathrm{GnRH}$-ant and GnRH-a was not significantly different (OR 0.86, 95\% CI 0.72 to $1.02, \mathrm{P}=0.085$ ), meaning that one could not identify significant differences with respect to the probability of live birth independently of the population studied, type of gonadotropin used for stimulation, or type of agonist protocol (fixed or flexible GnRH-ant regimen). The third study is an additional updated meta-analysis by $\mathrm{Al}$ Inany. This study showed that there was no significant difference following $\mathrm{GnRh}$ ant compared with $\mathrm{GnRh}$ agonist regimens (OR $0.86,95 \% \mathrm{CI}=0.69$ to 1.08 , $\mathrm{P}=0.20$ )in the live birth rate and in the ongoing pregnancy rate per woman randomized $(\mathrm{OR}=0.88,95 \% \mathrm{CI}=$ 0.77 to $1.00, \mathrm{P}=0.05)$.

\section{Conclusions of meta analysis}

Overall, these studies now demonstrate comparable efficacy and better safety of $\mathrm{GnRH}$ ant protocol than GnRh agonist protocol. Previous studies have shown a lower clinical and ongoing pregnancy rates for the $\mathrm{GnRh}$ antagonist protocols. In fact, these studies show some confounding variables from a methodological point of view: 1. data were pooled from patients with previously failed IVF attempts; 2. basal FSH, BMI, and duration of fertility were not stated; 3 . three type of antagonist protocol (single dose, flexible and fixed administration protocols), 4. GnRH-a treatment by either daily intranasal or subcutaneus administration, and 5. different starting dose of FSH were considered.

Moreover GnRH-ant were often used in cycles with an unfavourable prior outcomes, i.e. patients with advanced age and with a higher number of previously unfavourable cycles, thereby carrying a possible risk of introducing confounding factors.

As of now, we emphasize what has been suggested by Griesinger, that, "Perhaps GnRH antagonist is used as drug of second choice in IVF practice?" This Author, evaluating the data from the Germany IVF registry and stratifying the results by cycles rank, observed that the proportion of $\mathrm{GnRH}$-ant cycles increases from $23 \%$ in first treatment to $35 \%$ in fifth treatment and to $48 \%$ in tenth treatment. Engels et al., analyzing the data retrieved from the National Germany IVF registry demonstrated that $\mathrm{GnRH}$-ant are comparatively more often employed in higher ranks of treatment and that the proportion of older women is comparatively higher in antagonist cycles. Thus, they concluded that $\mathrm{GnRH}$-ant are currently often used as a second line medication or as first line treatment for patient with lower chances for pregnancy.

Sub-analysis of patients with equal demographic and clinical features resulted in similar pregnancy rates independent of whether GnRH agonist or antagonist was used

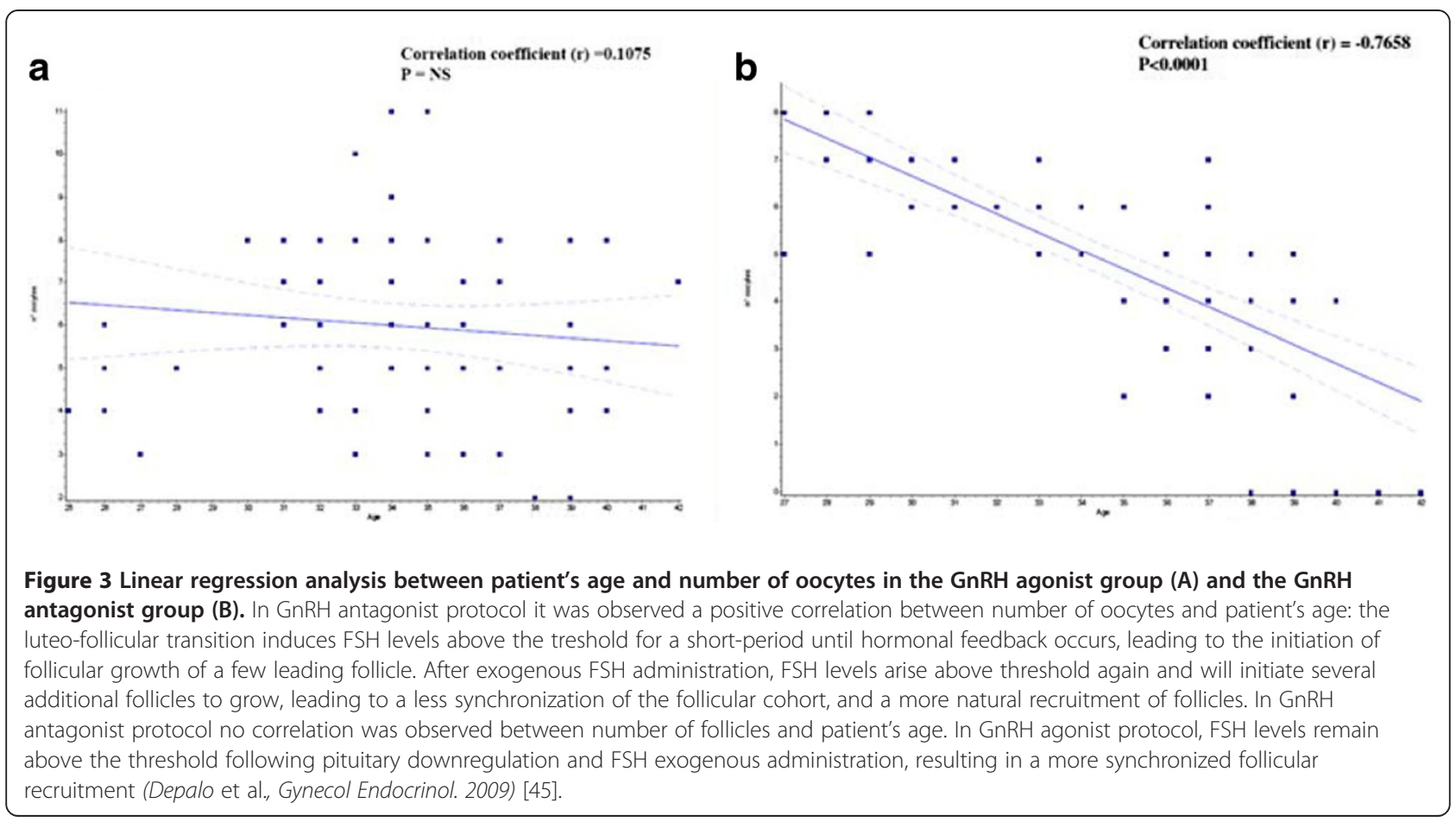


Table 1 Advantages and disadvantages of GnRH agonist protocols and GnRH antagonist protocols

\begin{tabular}{|c|c|c|c|c|}
\hline & GnRH Agonist long & GnRH Antagonist fixed & GnRH Antagonist flexible & $\begin{array}{l}\text { GnRH agonist short and } \\
\text { ultra-short }\end{array}$ \\
\hline Advantages & $\begin{array}{l}\text { A. Stable and low LH and P } \\
\text { levels throughout the } \\
\text { stimulation phaseB. } \\
\text { Suppression of endogenous } \\
\text { FSH levels leading to a } \\
\text { follicular cohort of all small } \\
\text { follilcles at the initiation of } \\
\text { FSH stimulation resulting in a } \\
\text { synchronized follicular } \\
\text { development }\end{array}$ & $\begin{array}{l}\text { A. Immediate, reversible } \\
\text { suppression of gonadotropin } \\
\text { secretion which avoids effects } \\
\text { related to the initial flare up } \\
\text { and subsequent down } \\
\text { regulationB. Initiation of the } \\
\text { IVF treatment in a normal } \\
\text { menstrual cycleC. } \\
\text { Endogenous inter-cycle FSH } \\
\text { rise rather than FSH } \\
\text { suppression, thus resulting in } \\
\text { a significant reduction in the } \\
\text { effective dosage and shorter } \\
\text { treatment, than with GnRHa }\end{array}$ & $\begin{array}{l}\text { A. Reduced dose of the } \\
\text { antagonist is neededB. The } \\
\text { cohort of follicles have more } \\
\text { time to develop thus leading } \\
\text { to a higher number of follicles } \\
\text { in mid-follicular phase }\end{array}$ & $\begin{array}{l}\text { A.The ovarian suppression is } \\
\text { not excessiveB. The initial } \\
\text { stimulation of the GnRH } \\
\text { receptors and consequent } \\
\text { secretion of endogenous } \\
\text { gonadotropins enhance the } \\
\text { effects of the exogenously } \\
\text { administered gonadotropins }\end{array}$ \\
\hline Disadvantagess & $\begin{array}{l}\text { A. More time counsuming } \\
\text { and complex stimulation } \\
\text { protocolsB. Acute stimulation } \\
\text { of gonadotropins and steroid } \\
\text { hormones due to the flare up } \\
\text { effectsC. Profound } \\
\text { hypoestrogenemia due to } \\
\text { downregulationD. Risk of } \\
\text { complications (OHSS) }\end{array}$ & $\begin{array}{l}\text { High intercycle endogenous } \\
\text { FSH concentrations inducing } \\
\text { secondary follicle recruitment } \\
\text { and leading to an } \\
\text { asynchronous follicular } \\
\text { development }\end{array}$ & $\begin{array}{l}\text { LH levels remain } \\
\text { unsuppressed during the early } \\
\text { follicular phase and enhance } \\
\mathrm{E}_{2} \text { production }\end{array}$ & $\begin{array}{l}\text { Flare up effects in mid- } \\
\text { follicular phase }\end{array}$ \\
\hline $\begin{array}{l}\text { Clinical } \\
\text { comments }\end{array}$ & $\begin{array}{l}\text { A. Increased number of } \\
\text { oocytes collectedB. Additional } \\
\text { pregnancy chances from } \\
\text { cryo-preserved embryosC. } \\
\text { Improvement in routine } \\
\text { patient treatment schedule }\end{array}$ & $\begin{array}{l}\text { A. More IVF cycles to be } \\
\text { carried out in a given } \\
\text { periodB. Starting stimulation } \\
\text { in patient scheduled for } \\
\text { antineoplastic treatments } \\
\text { (oocyte cryopreservation) }\end{array}$ & $\begin{array}{l}\text { It makes feasible to tailor } \\
\text { stimulation to patients' needs }\end{array}$ & $\begin{array}{l}\text { A. A microdose GnRHa flare } \\
\text { protocol is useful in poor } \\
\text { responders B. Several } \\
\text { microdoses of GnRHa in the } \\
\text { flare up protocols have been } \\
\text { tested to achieve } \\
\text { gonadotropin release and } \\
\text { avoid side-effects of the } \\
\text { classic flare up protocol }\end{array}$ \\
\hline
\end{tabular}

[44]. Thus, after critical appraisal of currently available studies using GnRH-ant it seems, that the differences in reported outcome measurements could be the consequence of the large variation of population included in the studies. In a RCT by our group, in which a strict inclusion criteria of patients was applied, it was shown that Implantation rate, clinical Pregnancy rate and miscarriage rates were similar in the $\mathrm{GnRH}$-antag regimens as well in GnRH-a long protocol. However a significantly higher number of oocytes and higher proportion of mature MII oocytes was retrieved per patient randomized, in the GnRH agonist group compared to the GnRH ant group. Moreover a significantly relationship was observed between patient's age and number of oocytes retrieved in antagonist group meaning that GnRH antag allows a more natural recruitment of follicles in the follicular phase in an ovary that has not been suppressed, whereas a better synchronization of the follicular cohort is observed in agonist treatment (Figure 3) [45] (Table 1).

More recently, a retrospective cohort review of first-time IVF cycles in good responders, has demonstrated that clinical pregnancy rates and live birth rates are similar utilizing either $\mathrm{GnRh}$ agonist or GnRh antagonist [46].

\section{Conclusions}

GnRH-ant regimen is effective in preventing a premature rise of $\mathrm{LH}$ and therefore results in a shorter and more cost-effective ovarian stimulation protocol compared to the long agonist protocol. However, there is difference in the synchronization of follicular recruitment and growth in the GnRH-a and GnRH-ant regimens, with better follicular growth and oocyte maturation seen with GnRH-a treatment [45].

The effect of elevated LH levels in follicular phase before GnRH-ant administration, has to be focused on. An optimization of the currently used stimulation protocol is needed with regard to timing of GnRH-ant administration, taking into account strategies for mild ovarian stimulation, making more patient friendly IVF protocols for patients who have to initiate radio-chemotherapy procedures.

Finally, several aspects of the GnRH-ant use needs to be further explored such as the direct effects of GnRHant on extra-pituitary tissues (i.e., corpus luteum, endometrium, ovary, embryo), and potential pharmacological differences among the existing compounds.

Competing interests

The authors declare that they have no competing interests. 


\section{Authors' contributions}

All authors have participated equally in the drafting of the manuscript. All authors have read and approved the final manuscript.

\section{Acknowledgements}

We thank dr K Jayakrishan for the correction of English language.

\section{Author details}

${ }^{1}$ Unit of Physiopathology of Human Reproduction and Gametes Cryopreservation, Department of Gynecology, Obstetric and Neonatolgy, University of Bari "Aldo Moro", Bari, Italy. ${ }^{2}$ KJK Hospital, Fertility Research Centre, Nalanchira- Trivandrum, Kerala, India. ${ }^{3}$ Section of Internal Medicine, Endocrinology, Andrology and Metabolic Diseases, Department of Emergency and Organ Transplantation (DETO), University of Bari "Aldo Moro", Bari, Italy.

Received: 18 September 2011 Accepted: 13 March 2012 Published: 13 April 2012

\section{References}

1. Caspi E, Ron-El R, Golan A, Nachum H, Herman A, Soffer Y, Weinraub Z: Results of in vitro fertilization and embryo transfer by combined long-acting gonadotropin-releasing hormone analog D-Trp-6-luteinizing hormone-releasing hormone and gonadotropins. Fertil Steril 1989, 51 (1):95-99.

2. Liu HC, Lai YM, Davis O, Berkeley AS, Graf M, Grifo J, Cohen J, Rosenwaks Z: Improved pregnancy outcome with gonadotropin releasing hormone agonist (GnRH-a) stimulation is due to the improvement in oocyte quantity rather than quality. J Assist Reprod Genet 1992, 9(4):338-344.

3. Zorn JR, Boyer P, Guichard A: Never on a Sunday: programming for IVF-ET and GIFT. Lancet 1987, 1(8529):385-386.

4. Daya S: Gonadotropin releasing hormone agonist protocols for pituitary desensitization in in vitro fertilization and gamete intrafallopian transfer cycles. Cochrane Database Syst Rev 2000, 2:CD001299.

5. Albuquerque LE, Saconato H, Maciel MC: Depot versus daily administration of gonadotrophin releasing hormone agonist protocols for pituitary desensitization in assisted reproduction cycles. Cochrane Database Syst Rev 2005, 1:CD002808.

6. Lorusso F, Depalo R, Selvaggi L: Relationship between gonadotropin releasing hormone agonist dosage and in vitro fertilization outcome. Gynecol Endocrinol 2004, 18(2):69-73.

7. Conn PM, Staley D, Harris C, Andrews WV, Gorospe WC, McArdle CA: Mechanism of action of gonadotropin releasing hormone. Annu RevPhysiol 1986, 48:495-513.

8. Rizk B, Smitz J: Ovarian hyperstimulation syndrome after superovulation using GnRH agonists for IVF and related procedures. Hum Reprod 1992, 7 (3):320-327.

9. Ben-Rafael Z, Lipitz S, Bider D, Mashiach S: Ovarian hyporesponsiveness in combined gonadotropin-releasing hormone agonist and menotropin therapy is associated with low serum follicle-stimulating hormone levels. Fertil Steril 1991, 55(2):272-275.

10. Ron-El R, Herman A, Golan A, Raziel A, Soffer Y, Caspi E: Follicle cyst formation following long-acting gonadotropin-releasing hormone analog administration. Fertil Steril 1989, 52(6):1063-1066.

11. Olivennes F, Frydman R: Friendly IVF: the way of the future?. Hum Reprod 1998, 13(5):1121-1124

12. Huirne JA, Homburg R, Lambalk CB: Are GnRH antagonists comparable to agonists for use in IVF?. Hum Reprod 2007, 22(11):2805-2813.

13. Al-Inany HG, Youssef MAFM, Aboulghar M, Broekmans FJ, Sterenburg MD, Smit JG, Abou-Setta AM: Gonadotrophin-releasing hormone antagonists for assisted reproductive technology. Cochrane Database of Systematic Reviews 2011, 11(5):CD001750.

14. Bodri D, Sunkara SK, Coomarasamy A: Gonadotropin-releasing hormone agonists versus antagonists for controlled ovarian hyperstimulation in oocyte donors: a systematic review and meta-analysis. Fertil Steril 2010, 95(1):164-169.

15. Humaidan P, Kol S, Papanikolaou EG: Copenhagen GnRH Agonist Triggering Workshop Group: GnRH agonist for triggering of final oocyte maturation: time for a change of practice?. Hum Reprod Update 2011, 4:510-524. Review.
16. Devroey P, Polyzos NP, Blockeel C: An OHSS-Free Clinic by segmentation of IVF treatment. Hum Reprod 2011, 26(10):2593-2597.

17. Oktay $\mathrm{K}$, Türkçüoğlu I, Rodriguez-Wallberg KA: GnRH agonist trigger for women with breast cancer undergoing fertility preservation by aromatase inhibitor/ FSH stimulation. Reprod Biomed Online 2010, 20(6):783-788.

18. Cavagna M, Dzik A: Depot GnRH-agonist trigger for breast-cancer patient undergoing ovarian stimulation resulted in mature oocytes for cryopreservation: a case report. Reprod Biomed Online 2011, 22(3):317-319.

19. von Wolff M, Montag M, Dittrich R, Denschlag D, Nawroth F, Lawrenz B: Fertility preservation in women-a practical guide to preservation techniques and therapeutic strategies in breast cancer, Hodgkin's lymphoma and borderline ovarian tumours by the fertility preservation network FertiPROTEKT. Arch Gynecol Obstet 2011, 284(2):427-435.

20. Olivennes F, Fanchin R, Bouchard P, de Ziegler D, Taieb J, Selva J: The single or dual administration of the gonadotropin-releasing hormone antagonist Cetrorelix in an in vitro fertilization-embryo transfer program. Fertil Steril 1994, 62(3):468-476.

21. Diedrich K, Diedrich C, Santos E, Zoll C, al-Hasani S, Reissmann T, Krebs D, Klingmüller D: Suppression of the endogenous luteinizing hormone surge by the gonadotrophin-releasing hormone antagonist Cetrorelix during ovarian stimulation. Hum Reprod 1994, 9(5):788-791.

22. Olivennes F, Alvarez S, Bouchard P, Fanchin R, Salat-Baroux J, Frydman R: The use of a GnRH antagonist (Cetrorelix) in a single dose protocol in IVF-embryo transfer: a dose finding study of 3 versus $2 \mathrm{mg}$. Hum Reprod 1998, 13(9):2411-2424.

23. Albano C, Smitz J, Camus M, Riethmüller-Winzen H, Van Steirteghem A, Devroey P: Comparison of different doses of gonadotropin-releasing hormone antagonist Cetrorelix during controlled ovarian hyperstimulation. Fertil Steril 1997, 67(5):917-922.

24. The ganirelix dose-finding study group: A double-blind, randomized, dose-finding study to assess the efficacy of the gonadotrophin-releasing hormone antagonist ganirelix (Org 37462) to prevent premature luteinizing hormone surges in women undergoing ovarian stimulation with recombinant follicle stimulating hormone (Puregon). Hum Reprod 1998, 13(11):3023-3031.

25. Wilcox J, Potter D, Moore M, Ferrande L, Kelly E: CAP IV Investigator Group: Prospective, randomized trial comparing cetrorelix acetate and ganirelix acetate in a programmed, flexible protocol for premature luteinizing hormone surge prevention in assisted reproductive technologies. Fertil Steril 2005, 84(1):108-117.

26. Olivennes F, Belaisch-Allart J, Emperaire JC, Dechaud H, Alvarez S, Moreau L: Prospective, randomized, controlled study of in vitro fertilization-embryo transfer with a single dose of a luteinizing hormone-releasing hormone (LH-RH) antagonist (cetrorelix) or a depot formula of an LH-RH agonist (triptorelin). Fertil Steril 2000, 73(2):314-320.

27. Ludwig M, Katalinic A, Banz C, Schröder AK, Löning M, Weiss JM, Diedrich K: Tailoring the GnRH antagonist cetrorelix acetate to individual patients' needs in ovarian stimulation for IVF: results of a prospective, randomized study. Hum Reprod 2002, 17(11):2842-2845.

28. Mochtar MH, Dutch Ganirelix Study Group: The effect of an individualized $\mathrm{GnRH}$ antagonist protocol on folliculogenesis in IVF/ICSI. Hum Reprod 2004, 19(8):1713-1718.

29. Al-Inany H, Aboulghar MA, Mansour RT, Serour Gl: Optimizing GnRH antagonist administration: meta-analysis of fixed versus flexible protocol. Reprod Biomed Online 2005, 10(5):567-570.

30. Kolibianakis EM, Albano C, Camus M, Tournaye H, Van Steirteghem AC, Devroey P: Initiation of gonadotropin-releasing hormone antagonist on day 1 as compared to day 6 of stimulation: effect on hormonal levels and follicular development in in vitro fertilization cycles. J Clin Endocrinol Metab 2003, 88(12):5632-5637.

31. Escudero E, Bosch E, Crespo J, Simón C, Remohí J, Pellicer: A Comparison of two different starting multiple dose gonadotropin-releasing hormone antagonist protocols in a selected group of in vitro fertilization-embryo transfer patients. Fertil Steril 2004, 81(3):562-566.

32. Kolibianakis EM, Zikopoulos K, Smitz J, Camus M, Tournaye H, Van Steirteghem AC: Administration of gonadotropin-releasing hormone antagonist from day 1 of stimulation in in vitro fertilization. Fertil Steril 2004, 82(1):223-226.

33. Cédrin-Durnerin I: Antagonist protocols: residual LH levels and the value of exogenous LH supplementation. J Gynecol Obstet Biol Reprod (Paris) 2004, 33(6 Pt 2):3S29-3S31. 
34. Cédrin-Durnerin I, Grange-Dujardin D, Laffy A, Parneix I, Massin N, Galey J, Théron L, Wolf JP, Conord C, Clément P, Jayot S, Hugues JN: Recombinant human LH supplementation during GnRH antagonist administration in IVF/ICSI cycles: a prospective randomized study. Hum Reprod 2004, 19 (9):1979-1984.

35. Aboulghar MA, Mansour RT, Serour Gl, Al-Inany HG, Amin YM, Aboulghar MM: Increasing the dose of human menopausal gonadotrophins on day of GnRH antagonist administration: randomized controlled trial. Reprod Biomed Online 2004, 8(5):524-527.

36. Merviel P, Antoine JM, Mathieu E, Millot F, Mandelbaum J, Uzan S: Luteinizing hormone concentrations after gonadotropin-releasing hormone antagonist administration do not influence pregnancy rates in in vitro fertilization-embryo transfer. Fertil Steril 2004, 82(1):119-125.

37. Baruffi RL, Mauri AL, Petersen CG, Felipe V, Martins AM, Cornicelli J, Cavagna M, Oliveira JB, Franco JG Jr: Recombinant LH supplementation to recombinant $\mathrm{FSH}$ during induced ovarian stimulation in the GnRH-antagonist protocol: a meta-analysis. Reprod Biomed Online 2007, 14 (1):14-25.

38. Huirne JA, van Loenen AC, Schats R, McDonnell J, Hompes PG, Schoemaker J, Homburg R, Lambalk CB: Dose-finding study of daily GnRH antagonist for the prevention of premature LH surges in IVF/ICSI patients: optimal changes in LH and progesterone for clinical pregnancy. Hum Reprod 2005, 20(2):359-367.

39. Kolibianakis EM, Collins J, Tarlatzis BC, Devroey P, Diedrich K, Griesinger G: Among patients treated for IVF with gonadotrophins and $\mathrm{GnRH}$ analogues, is the probability of live birth dependent on the type of analogue used? A systematic review and meta-analysis. Hum Reprod Update 2006, 12(6):651-671.

40. Griesinger G, Kolibianakis EM, Venetis C, Diedrich K, Tarlatzis B: Oral contraceptive pretreatment significantly reduces ongoing pregnancy likelihood in gonadotropin-releasing hormone antagonist cycles: an updated meta-analysis. Fertil Steril 2010, 94(6):2382-2384.

41. Kosmas IP, Zikopoulos K, Gorgiou I, Paraskevaidis E, Blockeel C, Tournaye H, Van Der Elst J, Devroey P: Low-dose HCG may improve pregnancy rates and lower OHSS in antagonist cycles: a meta-analysis. Reprod Biomed Online 2009, 19(5):619-630.

42. European and Middle East Orgalutran Study Group: Comparable clinical outcome using the GnRH antagonist ganirelix or a long protocol of the GnRH agonist triptorelin for the prevention of premature LH surges in women undergoing ovarian stimulation. Hum Reprod 2001, 16(4):644-651.

43. Huirne JA, Lambalk CB: Gonadotropin-releasing-hormone-receptor antagonists. Lancet 2001, 358(9295):1793-1803.

44. Engel JB, Griesinger G, Schultze-Mosgau A, Felberbaum R, Diedrich K: GnRH agonists and antagonists in assisted reproduction: pregnancy rate. Reprod Biomed Online 2006, 13(1):84-87.

45. Depalo R, Lorusso F, Palmisano M, Bassi E, Totaro I, Vacca M, et al: Follicular growth and oocyte maturation in GnRH agonist and antagonist protocols for in vitro fertilisation and embryo transfer. Gynecol Endocrinol 2009, 25(5):328-334.

46. Johnston-MacAnanny EB, DiLuigi AJ, Engmann LL, Maier DB, Benadiva CA, Nulsen JC: Selection of first in vitro fertilization cycle stimulation protocol for good prognosis patients: gonadotropin releasing hormone antagonist versus agonist protocols. J Reprod Med 2011, 56(1-2):12-26.

\section{Submit your next manuscript to BioMed Central and take full advantage of:}

- Convenient online submission

- Thorough peer review

- No space constraints or color figure charges

- Immediate publication on acceptance

- Inclusion in PubMed, CAS, Scopus and Google Scholar

- Research which is freely available for redistribution

Submit your manuscript at www.biomedcentral.com/submit 